\title{
INFECTED URACHAL CYST - A RARE CASE REPORT
}

Gopalakrishna K. H'1 Rajashekar K. A2 , Karthik B ${ }^{3}$

\section{HOW TO CITE THIS ARTICLE:}

Gopalakrishna K. H, Rajashekar K. A, Karthik B. "Infected Urachal CYST - A Rare Case Report". Journal of Evolution of Medical and Dental Sciences 2014; Vol. 3, Issue 47, September 25; Page: 11457-11462,

DOI: $10.14260 /$ jemds/2014/3499

ABSTRACT: An infected urachal cyst is one among the spectrum of presentations of urachal pathology, which are rare in adulthood. It occurs in a 2: 1 male to female ratio with an incidence of about $2 \%$ in adults. Patients with infected urachal cysts can present with a wide range of symptoms, most commonly abdominal pain, fever, umbilical discharge and the feeling of a midline mass Treatment is initiated with intravenous antibiotics followed by complete excision of the umbilicovesical tract including a cuff of bladder.

KEYWORDS: Urachus, Sinus, Cyst, Vitellointestinal duet.

INTRODUCTION: An infected urachal cyst is one of a spectrum of presentations of urachal pathology, all of which are rare in adulthood. Usually urachal anomalies remain asymptomatic unless there are complications such as infection, lithiasis and malignant degeneration. Patients tend to present in an heterogeneous pattern making diagnosis difficult. It occurs in a 2:1 male to female ratio with an incidence of about $2 \%$ in adults ${ }^{1}$. Here we present a case report on Infected Urachal Cyst in an adult.

CASE PRESENTATION: A 22 year old male patient was referred from a private hospital with a history of Fever for 15 days, low grade, intermittent type and pain abdomen since 15 days insidious in onset, gradually progressive present around the umbilicus, it was throbbing type of pain with burning sensation, non-radiating, used to relieve on medication and also discharge through the umbilicus since 12 days, serous in nature, scanty in amount, and foul smelling. As the day progressed it was admixed with blood which later turned into yellowish tinged, following this the patient developed a swelling in the umblical region about $1 \times 2 \mathrm{~cm}$, pinkish colour, later turned to blackish colour after a day. While excising this swelling, it spontaneously ruptured and pus-filled discharge oozed out, following which the patient was referred here.

On examination the patient's general condition were fair, He was febrile, pulse -96bpm regular, good volume. BP $-110 / 70 \mathrm{mmhg}$. Cardiovascular system, respiratory system and central nervous system were found to be normal.

Per abdomen examination with the patient in supine position revealed:

- On Inspection: a discharging sinus through the umbilicus measuring around $1 \times 2 \mathrm{~cm}$ was present. The surrounding skin is red in colour. There was presence of yellowish foul smelling discharge through the sinus.

- On Palpation: There was local rise of temperature and tenderness around the umbilicus. A discharging sinus through the umbilicus measuring of around $1 \mathrm{x} 2 \mathrm{~cm}$ was present.

Routine Blood investigations were found to be within normal limits. Ultra sound abdomen revealed infected urachal cyst which was presenting as umbilicus sinus. 


\section{CASE REPORT}

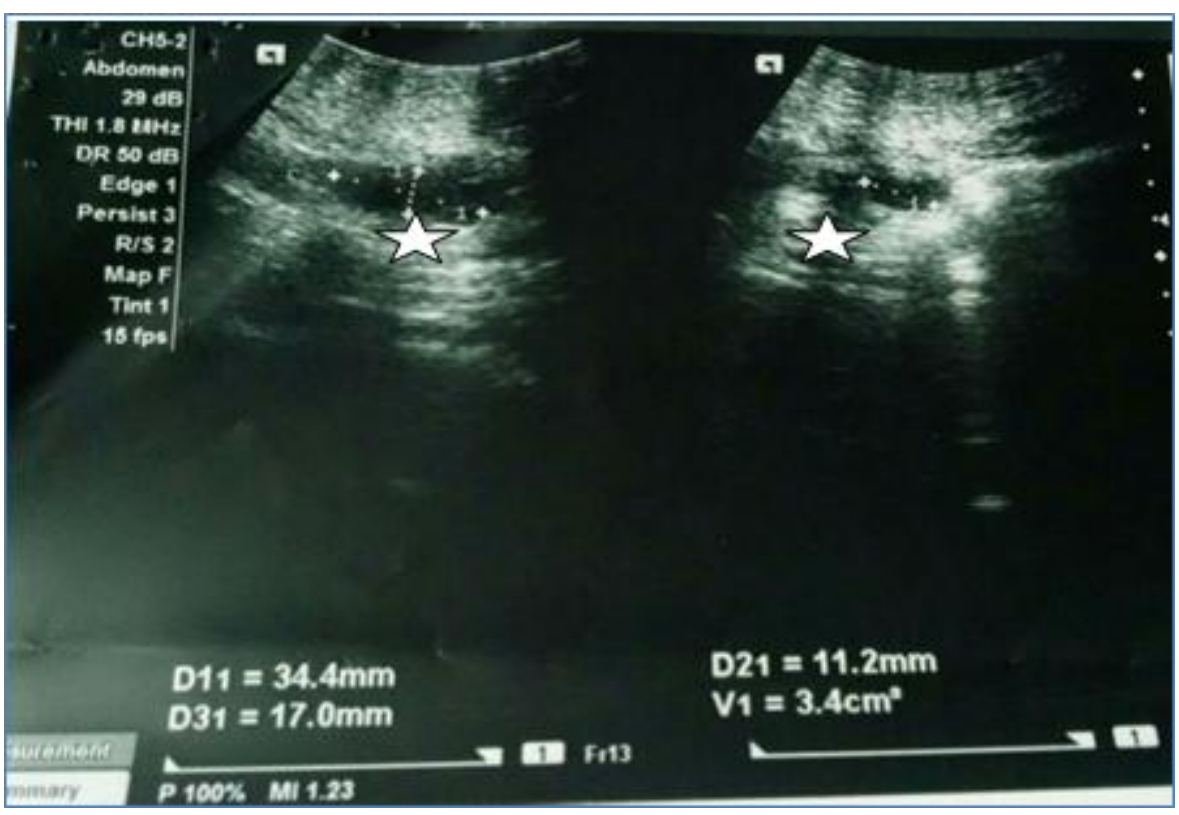

Fig. 1: $\sum$ USG Abdomen showing Infected Urachal cyst

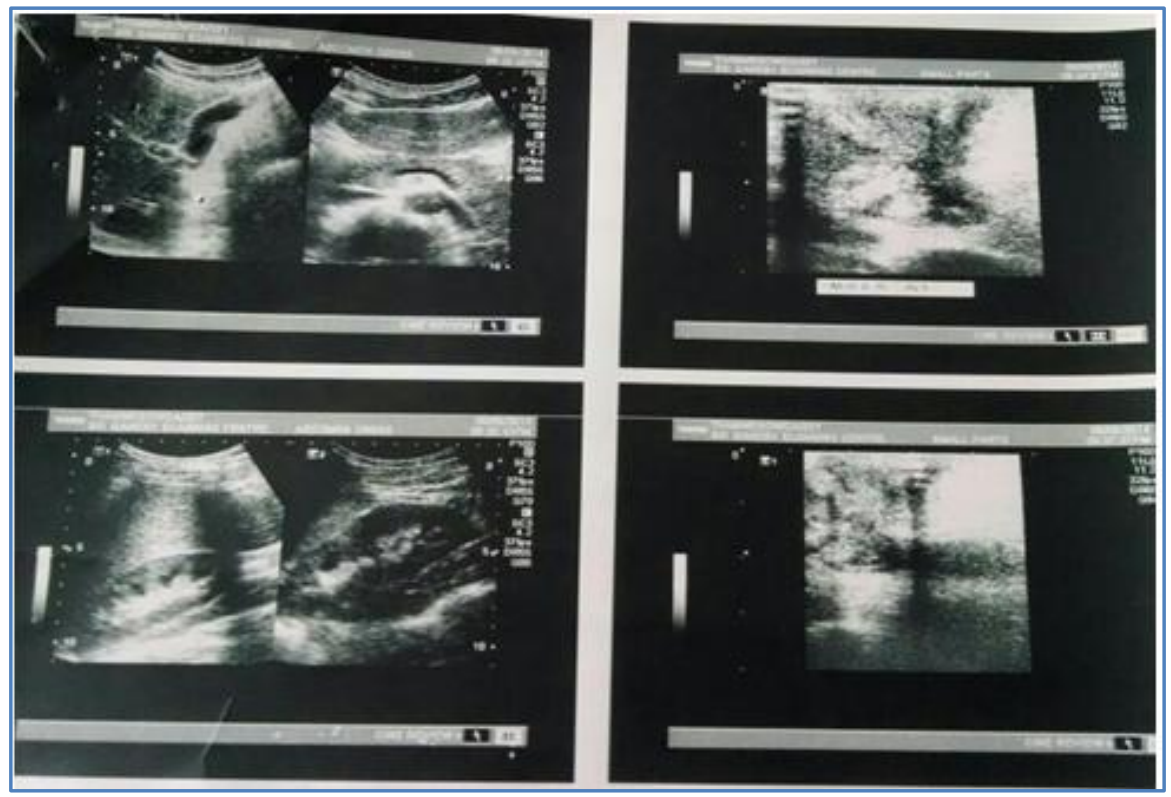

Fig. 2: USG abdomen showing Umbilical sinus

The patient was treated with Inj cefotaxime 1gm IV BDx7days, Inj metronidazole $500 \mathrm{mg}$ IV TIDx9days, Inj Tramadol IM BD, Inj Ranitidine 150 mg BD x3day, Tab Aceclo sp BD, Tab Ranitidine BD, Tab Enractin, Tab Cefexime oz BD x 7daysTab, following which the discharge subsided. 


\section{CASE REPORT}

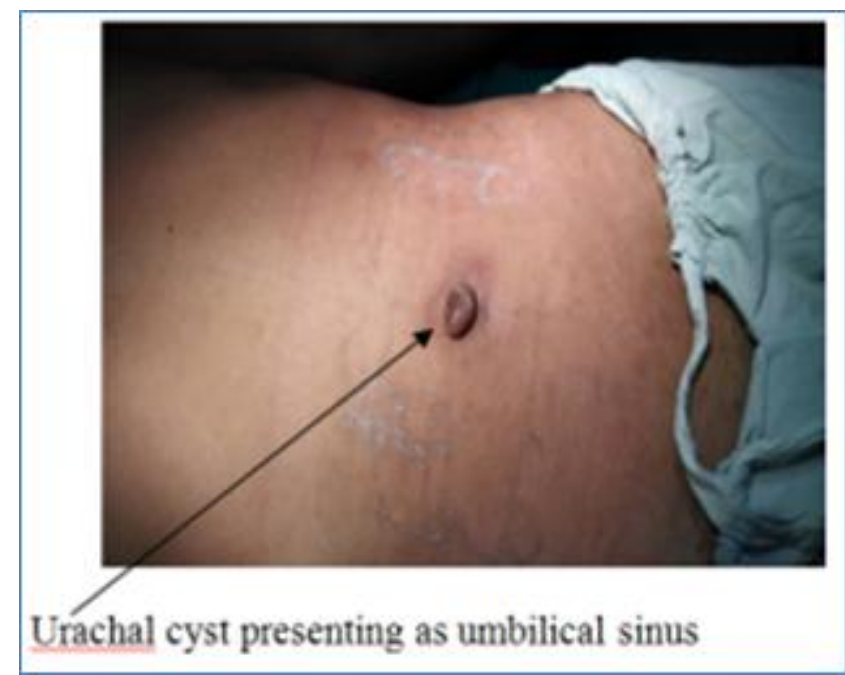

Figure 3

Procedure done - Excision of the urachal cyst

Patient placed in supine position after giving Sub Arachnoid Block, parts painted and draped. Methylene blue dye injected into the sinus, a " $\mathrm{T}$ " shaped incision was made including the umbilicus and extended below, and the sinus was excised, and the specimen sent for Histo Pathological Examination.

Per operatively: No connection was found between the cyst and the bladder.

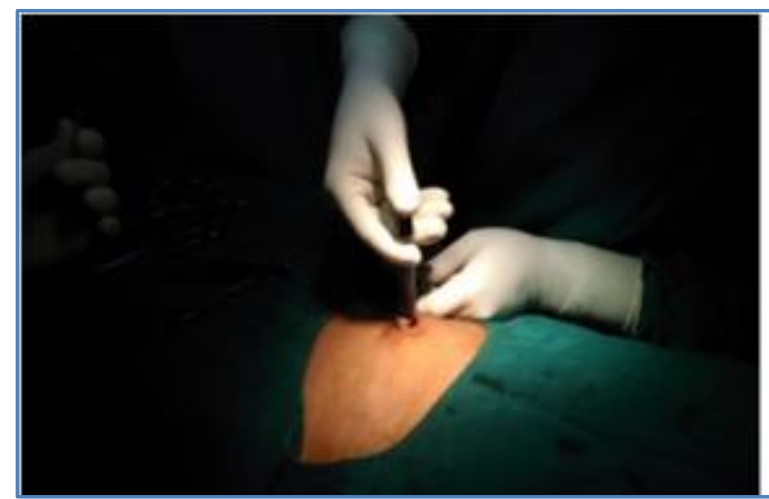

Fig. 4: Methylene blue dye being injected into the sinus

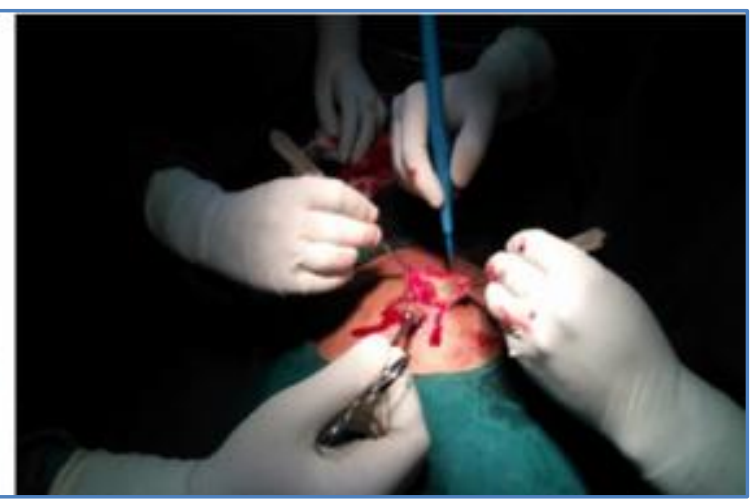

Fig. 5: T shaped incision with excision of sinus

Abdomen was closed in layers, after placing corrugated drain insitu. 


\section{CASE REPORT}

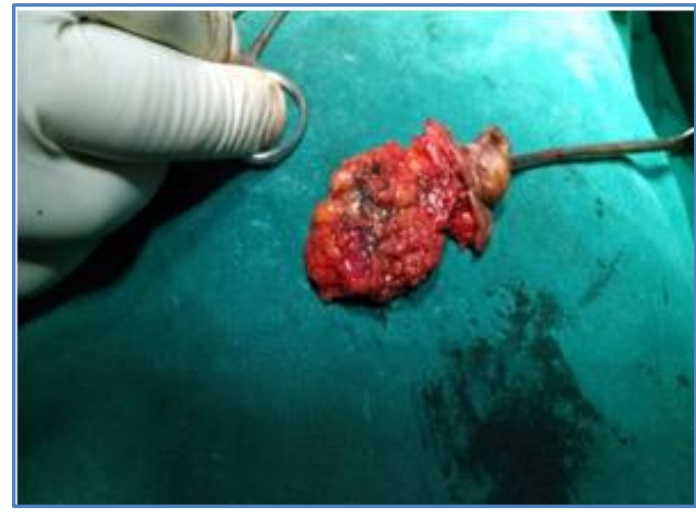

Fig. 6: Excised Sinus

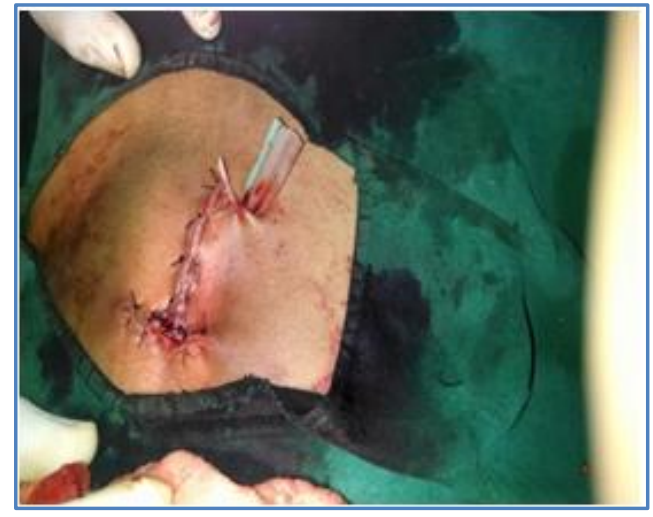

Fig. 7: Abdomen closed with corrugated drain insitu

Post operative period was uneventful, drain removed on third post operative day and suture removed on 7 th post operative day and the patient was discharged.

Histopathological examination of the specimen Revealed features suggestive of chronic non specific inflammatory sinus consistent with inflamed urachal cyst.

\section{Microscopy:}

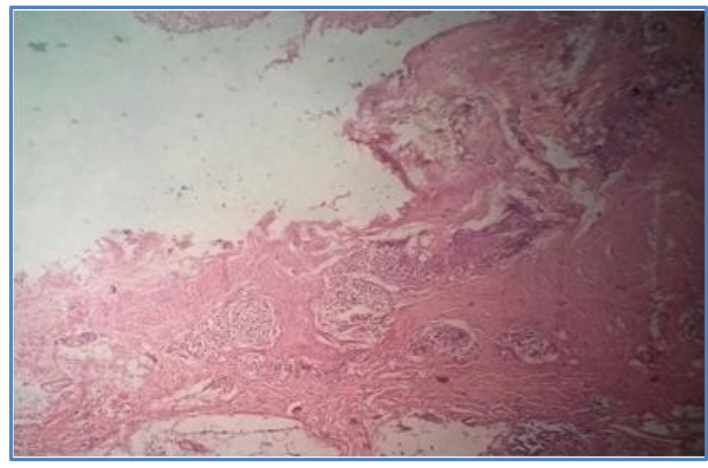

Fig. 8

Fig. 8: Features suggestive of nonspecific inflammatory sinus with features suggestive of inflamed urachal cyst.

DISCUSSION: The urachus developmentally is the upper part of the bladder which arises from the ventral part of the cloaca and allantois ${ }^{2}$. Further descent of the bladder at $4^{\text {th }}-5^{\text {th }}$ month of gestation into the fetal pelvis results in the formation of the urachal canal, which later forms a fibrous tract, extending from the anterior bladder wall towards the umbilicus, as the median umbilical ligament, within the space between the peritoneum and trasversalis fascia. ${ }^{3}$ 
Urachal anomalies may be present in $2 \%$ of general population and they are classified into four anatomical categories-patent urachus-50\%, urachal diverticulum -3to5\%, umbilical urachal sinus-15\%, and urachal cyst - $30 \% .4$

Urachal anomalies usually remain asymptomatic unless they are complicated with infection, lithiasis and malignant degeneration. Urachal cysts present in a variety of clinical presentations including recurrent urinary tract infections, macroscopic hematuria, and hypogastric midline tenderness often associated with a mass, umbilical discharge, and also peritonitis. In children an urachal cyst is the common presentation whereas in adults infected urachal sinuses are seen more frequently ${ }^{5}$. Morbidity is related to infection and possibly, late occurring malignant changes. Following gross infection, the cyst often expands forming a Pyourachus which can then establish communication with the umbilicus, bladder, bowel or even rupture intraperitoneally. Bacteria commonly implicated in the causation of infection include Staphylococcus, E.coli, Pseudomonas, streptococcus, and pseudomonas aeruginosa. ${ }^{6}$. Though rare, the most common malignancy associated with an urachal cyst is Adenocarcinoma of the urachal remnant especially at the apex of the bladder. ${ }^{7}$

Owing to the nature of the condition, a high degree of clinical suspicion followed by radiographic evaluation by ultrasonography, CT and MRI is essential for diagnosing an urachal cyst or abscess, the most common diagnostic finding includes, a midline, cystic, extraperitoneal swelling located between the bladder and the umbilicus ${ }^{8}$. Abdominal tomography can accurately detect the pyourachus which shows imaging of a mass located deep to the rectus abdominis between the bladder and the umbilicus with a conical shape, peripheral inflammatory changes in the surrounding tissues, and intraperitoneal fluid ${ }^{9}$. Retrograde cystography can also be helpful in characterizing bladder patency in advanced cases.

Treatment is initiated with intravenous antibiotics followed by complete excision of the umbilicovesical tract including a cuff of bladder. For lesions not communicating with the bladder, conservative excision of the remnant cyst is adequate. Removal of all urachal elements is necessary to avoid the possible recurrence of the cyst or of adenocarcinoma of the retained elements. ${ }^{10}$

The traditional treatment of an infected urachal cyst is composed of a two stage approach - An incision and then drainage of the infected cyst followed by secondary excision, however, a laparoscopic approach is also an attractive alternative as it is as effective and as safe as the open operation with the additional advantages of decreased hospital stay, analgesic requirement, and convalescence ${ }^{11}$. In cases where a pre-operative diagnosis has been made, a staged approach with antibiotics followed by surgery has been recommended.

Delay in treatment may have serious consequences as complications include sepsis fistula formation and rupture leading to peritonitis. Necrotizing fasciitis has also been reported as a rare complication of an infected urachal cyst. ${ }^{12}$

CONCLUSION: Urachal anomalies are in adults, presentation is atypical, and therefore a high index of suspicion is required in order to achieve a diagnosis. A triad of lower midline mass, umbilical discharge and sepsis is suggestive, although MRI confirms the diagnosis and defines the surrounding anatomical relationship. Complete surgical excision is the treatment of choice due to the risk of malignant transformation. We recommend a 2 stage treatment with a combination of broad spectrum antibiotics or incision and drainage, followed by interval excision after resolution of sepsis. 


\section{REFERENCES:}

1. Ashley R A, Inman B A, Routh J C, Rohlinger A L, Husman D A, Kramer S A. Urachal anomalies: a longitudinal study of urachal remnants in children and adults. Journal of Urology. 2007; 178: 1615-1618.

2. Begg R C. The urachus: its anatomy, histology and development. Journal of Anatomy. 1930; 64: 170-183.

3. Khalid Qureshi, David Maskell, Colin McMillan, Chandana Wijewardena. An infected urachal cyst presenting as an acute abdomen - A case report. Int J Surg Case Rep. 2013; 4(7): 633-635.

4. Lan Bickle, Yuranga Weerakkody, Congenital urachal remnant abnormalities, www.radiopaedia.org

5. Yoshimura K, Toki K, Koide T, Ashino N, Maki T. Infantile granulomatous urachal abscess with acute localized peritonitis and appendicitis. International J Urology. 1(4): 352, 1994.

6. Suita S, Nagasaki A. Urachal remnants. Seminars in Pediatric Surgery. 5(2)107-115, 1996.

7. Dandekar NP, Dalal AV, Tongaonkar HB, Kamat MR. Adenocarcinoma of the bladder. European J. of Surg. Oncology. 23(2)157-160, 1997.

8. Holten I, Lomas F, Mouratidis B, Malecky G, Simpson E. The ultrasonic diagnosis of urachal abnormalities. Australasian Radiology. 40(1)2-5, 1996.

9. Herman TE, Shackelford GD. Pyourachus: CT manifestations. Journal of Computer Assisted Tomography. 19(3): 440-3, 1995.

10. C Perry, B Phillips. Clinical Management of Urachal Cysts. The Internet Journal of Urology. 2000 Volume 1 Number 1

11. Stone NN, Garden RJ, Weber H. Laparoscopic excision of an urachal cyst. Urology. 45(1)161164, 1995.

12. Testerman G.M. Necrotizing fasciitis due to an infected urachal cyst in an adult. Southern Medical Journal. 2010; 103: 1066-1067.

\section{AUTHORS:}

1. Gopalakrishna K. H.

2. Rajashekar K. A.

3. Karthik B.

\section{PARTICULARS OF CONTRIBUTORS:}

1. Assistant Professor, Department of General Surgery, MIMS, Mandya.

2. Assistant Professor, Department of General Surgery, MIMS, Mandya.

3. Senior Resident, Department of General Surgery, MIMS, Mandya.

\section{NAME ADDRESS EMAIL ID OF THE} CORRESPONDING AUTHOR:

Dr. Gopalakrishna K. H, Assistant Professor, Department of General Surgery, MIMS, Mandya.

Email: drgopala566@gmail.com

Date of Submission: 16/09/2014.

Date of Peer Review: 17/09/2014.

Date of Acceptance: 18/09/2014.

Date of Publishing: 25/09/2014. 